increased and endothelial degeneration of its vessels is revealed. These morphological changes are restored to the normal condition by the osmotherapy.

2) Formation of collagenous fibers in the capsule of hematoma is promoted by the osmotherapy.

\title{
84. An Angiographical Study on Acute Severe Head Injuries
}

\author{
With Special Reference to the Indication \\ for the Operation and the Prognosis \\ Takachika KajuUra, Yoshiaki Ueda, \\ Mitsuyoshi Enoki, Akira Kitagawa \\ Department of Neurosurgery, Osaka Policeman Hospital \\ Takahiro NArTo \\ 2nd Department of Surgery, Nara Medical University
}

It has been emphasized that the prognosis of the acute head injuried patient depends upon the severity of the head injury, the age of the patient, the general condition before having head injury and the appropriate treatment for its patient. Our report concerns the relationship of the various findings on anterior-posterior projection film of CAG at the acute stage of the head injury and the prognosis of the patients who are diagnosed as the localized mass lesion associated with the abnormality of vascularity on carotid angiogram, and had emergency operations which include the internal or external decompressed procedures.

A series of 23 cases of severe head injuries has recently been experienced in our clinic. Seventeen patients were male and the others were female. The ages of these patients ranged between 1 and 73 years at the time of operation. The operative mortality of our 23 cases is 39.1 per cent which includes 9 cases of death, and the other hand, there were two cases of the akinetic mutisum among the alive cases after operation.

1) Circulation time was checked on CAG 5 seconds after injection of the contrast media. There was the only one case of the death in 9 cases without the delayed circulation time. Among the cases which showed capillary phase on CAG within 5 seconds after injection there was a death case out of 7 cases. No patients were survived in 7 cases which capillary phase on CAG was visualized at more than five seconds after the injection of the contrast media.

2) The shift of the anterior cerebral artery; Eight out of 11 cases were died as having more than $10 \mathrm{~mm}$ shift of the anterior cerebral artery on A-P view of CAG

3) The deviation of the middle cerebral artery; We made the criteria on devia- 
tion of the middle cerebral artery based on normal angiographic sylvian point of Taveras et al. The abnormal value was settled on having deviation of $5 \mathrm{~mm}$ in length vertically and $2 \mathrm{~mm}$ in length horizontally from Taveras' normal sylvian point. Nine of 17 cases with these abnormal values were died.

4) Shape of the posterior knee (Cl) of internal carotid artery. Among 5 cases whose $\mathrm{Cl}$ showed the upright position on $\mathrm{CA} \mathrm{G}$, three cases were died after operation. No survivor was noted after operation in 6 cases whose $\mathrm{Cl}$ was declined much more medially.

5) The deviation of the anterior communicating artery. There were nine deaths of 10 cases with the deviation of A-C artery, and unfortunately the only one survived case disclosed an akinetic mutisum.

In conclusion our results of the circulation time on CAG were the same as the earlier description of many authors. The deviation of $\mathrm{Cl}$ and anterior communicating artery, however, might be suggesting the anatomical deformity of brain stem on A-P view of CAG. It may emphasize that the faster anatomical deformity of the brain stem the worse the prognoses of these patients. The fact may be recognized that the patients who shows brain stem syndrome in there clinical courses would have the worse prognosis. We could say that if these patients would have emergency operation at acute stage it is very difficult to relieve their lives in cases who show the marked deviation of $\mathrm{Cl}$ and anterior communicating artery.

\title{
Discussion to 84 Angiographic Area Index of the Avascular Area of the Sinus Epidural Hematoma and its Value in the Prognosis
}

\author{
Yuji Mryazaki, Ikuro Takada and Hideyuki Ando \\ Department Neurological Surgery, Sapporo Medical College and Hospital
}

There are many reports on the relationship between the cerebral angiographic findings and the prognosis in the cases of traumatic intracranial hematoma. However, it is hard to get the information about the prognosis from the cerebral angiogram related with the size of avascular area and grade of dislocation of main cerebral arteries, even in cases of epidural hematoma who is accompanying none or a little brain contusion and whoes prognosis is affected by the amount of blood clots mainly. Moreover, in the cases of acute subdural hematoma, it is more difficult to estimate the prognosis from cerebral angiogram alone.

On the other hand, in the cases of sinus epidural hematoma located in the midline, the thickness of the epidural heamatoma is able to measure by the area of the avascular area in the lateral film of venous phase of angiogram and this point is quite 